\title{
Energy and Load Aware Routing Protocol for Internet of Things
}

\author{
S.Sankar ${ }^{1}$, P.Srinivasan ${ }^{2}$ \\ ${ }^{1}$ Research Scholar, School of Computer Science and Engineering, VIT University, Vellore-632014, Tamilnadu, India; \\ ${ }^{2}$ Associate Professor, School of Information Technology and Engineering, VIT University, Vellore-632014, \\ Tamilnadu, India
}

\begin{tabular}{l} 
Article Info \\
\hline Article history: \\
Received Nov 28, 2017 \\
Revised Feb 18, 2018 \\
Accepted Mar 11, 2018 \\
\hline Keyword: \\
Enery Efficiency \\
Internet of Things \\
IPv6 Routing Protocol for Low- \\
Power and Lossy Networks \\
Load
\end{tabular}

\section{Corresponding Author:}

P.Srinivasan

Associate Professor,

School of Informationa and Technology,

VIT University, Vellore-632014, Tamilnadu, India;

Email: srinivasan.suriya@vit.ac.in

\begin{abstract}
Maximizing the network lifetime is one of the major challenges in Low Power and Lossy Networks (LLN). Routing plays a vital role in it by minimizing the energy consumption across the networks through the efficient route selection for data transfer. IPv6 Routing Protocol for Low Power and Lossy Networks (RPL) is a IETF standardized IPv6 routing protocol for LLN. In this paper, we propose Energy and Load aware RPL (EL-RPL) protocol, which is an enhancement of RPL protocol. It uses a composite metric, calculated based on expected transmission count (ETX), Load and battery depletion index (BDI), for the route, selection. The COOJA simulator is used for performance evaluation. EL-RPL is compared with other similar protocols RER(BDI) RPL and fuzzy logic based RPL (OF-FL RPL). The simulation result shows that the EL-RPL improves the network lifetime by 8$12 \%$ and packet delivery ratio $2-4 \%$.
\end{abstract}

Copyright (c) 2018 Institute of Advanced Engineering and Science. All rights reserved.

\section{INTRODUCTION}

Internet of Things (IoT) is an emerging research area of last few years and it is a future internet technology. IoT enables the machine-to-machine communication that able to exchange the data between them and take the decision accordingly without involvement of human [1-2]. Nowadays, IoT system is involved in various field and the application areas such as smart home, wearable, smart grid, smart city, connected car, smart retail, smart farming, smart supply and etc.,. IoT is a new paradigm that introduced a new way of communication and it is denoted as Low Power and Lossy Networks (LLN) [3]. In LLN, the nodes are highly resource constrained i.e, low power, processing and memory capacity. The existing routing protocols namely on-demand distance vector (AODV), optimized link state routing(OLSR), open shortest path first (OSPF) and intermediate system to intermediate system (IS-IS) for ad-hoc network that unable to fulfill the requirements of LLN. So, IETF working group is standardized an IPv6 Routing protocol for Low power and Lossy networks (RPL) for LLN [4-5].

RPL is a distance vector and source routing protocol and it follows the Destination oriented Directed Acyclic graph (DODAG). The top of the node is represented as root node. The edges directed towards the root called upward routing and edges are far away from root called downward routing. RPL protocol generates more than one RPL instances in a network and each instance contains more than one DODAG. The entire RPL instance maintains in separate RPL instance ID or DODAG ID. Each node has own IPv6 address. In LLN, the top of the node acts as border router and remaining entire node acts as host node of DODAG. The host node gets the address from root ID prefixes. The border router or root node maintains the entire 
network information in a routing table. RPL has two types of nodes i.e, storing and non- storing mode. The storing mode can collect and forward the data to other node. The non-storing mode simply forwards the data to other node. In RPL, DODAG construction process is used for three control messages namely, DODAG Information Solicitation (DIS), DODAG Advertisement Object (DAO), DODAG Advertisement ObjectAcknowledgement (DAO-ACK). The participant node sends the willingness to DODAG or DODAG sends the DIO control messages to all the participants. Then the participant node sends the DAO control messages with in trickle time. Finally, the DODAG sends the DAO-ACK confirmation messages to all participants [6-7].

The proposed work main objective is to maximize the network lifetime by minimizing the node energy consumption. The contribution of this work is to introduce a combination of ETX, Load and BDI based composite metric in RPL. This composite metric follows the minimizable property. DODAG sends DIO control messages to all participant nodes. The participant node selects the best parent from DODAG rank. The rank calculates from minimum value of the composite metric in the DODAG. Finally, sender or participant node sends the data to DODAG root towards the best parent in the DODAG. Thus, it improves the packet delivery ratio, reduce the traffic load and improve network lifetime.

\subsection{Problem Statement}

RPL is designed and implemented with variaous features such as delay, self healing, loop-free topology and load balance. Nevertheless, they are not considered at a time, these metrics such as Load, Residual Energy and Link quality and this imbalance is a significant limitation in RPL. This problem creates more impact to drain the node energy in near to the sink node and as well as intermediate node. The proposed routing protocol (EL-RPL) balances the energy and load among the network nodes and it avoids the bottle neck in near to the sink node and also intermediate node.

\subsection{RPL Protocol Based Related Works}

In this section briefly discuss energy efficient RPL protocol issues in LLN. The composite metric indicates the composition of more than one routing metric in DODAG. The composite metric provides the requirement of convergence, optimality, and loop-freeness in LLN.

Zahariadis et'al [8] proposed a design guideline for routing metrics composition in LLN and this document is standardized by IETF. It is clearly stated the properties, rules and requirement of composite metrics in LLN. The composition of the primary metric can be combined additive and lexical manner. Additive property is suitable for composition of primary metric consists of either minimizable or maximization property. Fuzzy logic is suitable for composition of single metric consists of both minimizable and maximization. Lexicographic property is suitable for composition of single metric and it is inspected first metric and only if possible path have equal value then it considers the next metric from the composition.

Oana Iova et' al [9] proposed expected lifetime (ELT) as routing metric and it estimates the bottleneck of the network node. It mainly focused on network holes near the sink node. It is considered the factors such as traffic and link reliability. ELT metric avoids the early node battery depletion during the data transmission and network holes near sink node.

Ali Hassan et'al [4] proposed an improved routing metrics for RPL protocol in LLN. This composite metric based on the combination of residual energy (RES), expected transmission count (ETX) and battery depletion index (BDI). It improves the network lifetime and reduces the battery depletion. However, this work is not given the preferences to link quality (ETX).

Patrick-Olivier Kamgueu et' al [10] proposed a fuzzy inference mechanism based composite metric. This composite metric based on the combination of delay, ETX and residual energy. It mainly concentrated on quality of service in LLN. It minimizes the energy consumption and improves the quality of service.

Panagiotis Karkazis et'al [11] evaluated the routing metric composition to improve the Quality of Service (QoS) in LLN. It focused on three things. a). it introduced a composite metric from the single metric such as Remaining energy (RE), Expected transmission count (ETX), Packet forwarding indication (PFI). b). It provided better QoS and it proved the efficiency using routing algebra. c). it achieved better performance and it is provided the optimal loop- free paths.

\section{SYSTEM MODEL}

Figure 1 indicates the IoT low power and lossy networks system model. Thousands of sensor nodes are connected to the Border Router (BR) via mesh network. The BR or Low power Border router (LBR) connects the LLN edge devices in to both public or private internet and various application servers are 
connected in the internet. In LLN, the edge devices are generating the data and send to the border router and various applications access those sensor data through internet. In LLN, we can perform the upward and downward routing. If one device wants to communicate with other device, that node sends the data to border router and it forwards the data to corresponding destination through downward routing $[12,13]$.

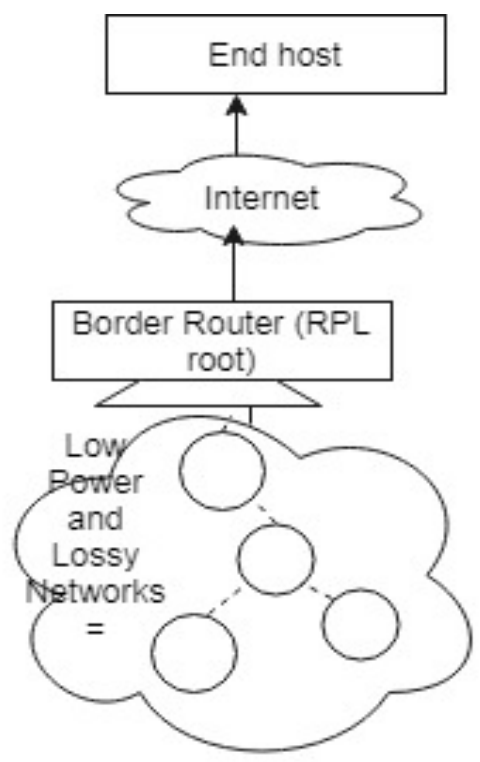

Figure 1. Low Power and Lossy Networks System Model

\section{THE PROPOSED EL RPL PROTOCOL}

In this paper, we propose energy and load aware composite routing metric (EL-RPL). EL-RPL is a composite routing metric and it is based on the combination of load, battery depletion index (BDI) and expected transmission count (ETX). In EL-RPL, the participant node selects the best parent among the preferred parent based on DODAG rank. The rank calculates from objective function and rank increase. Each node and link associates with Load, BDI and ETX metrics in LLN. This composite metric improves the network lifetime.

\subsection{Metrics of Interest}

\subsubsection{Expected Transmission Count (ETX)}

Expected Transmission Count is a link metric and it is used to predict the link quality based on transmission and including retransmission. The ETX metric formula calculates from Equation (1) and (2).

\section{Link ETX}

Link ETX represents the forward and reverse data delivery of link. The forward data delivery (df) represents the probability that a data packet, successfully arrives at the recipient. Reverse data delivery (dr) represents the probability that the ACK packet received successfully from the recipient [14]. The link ETX calculates from Equation (1).

$$
\operatorname{ETX}(N i)=\frac{1}{d f * d r}
$$

\section{Route ETX}

Route ETX uses to find link quality of particular path Px. The Route ETX calculates from Equation

$$
\operatorname{ETX}(P x)=\sum_{i=1}^{n} \operatorname{ETX}(N i)
$$




\subsubsection{Load}

Network data traffic is an amount of data transfer across the network at given amount of time. Load balance is a technique and it is used to balance the traffic across network. It is mainly concentrated on number of child present in each parent node [15]. The participant node selects the parent node based on less number of child accumulated parent node in the DODAG.The traffic load calculates from equation (3) and (4). If the number of children increases in a parent node in the DODAG, EL-RPL reconstructs the DODAG.

\section{a. To calculate the Load}

In EL-RPL, load of Path(x) calculation is based on the cumulative of node traffic or child set.

$$
\operatorname{Load}(\operatorname{Path}(x))=\sum_{M=1}^{n} \text { Node__TrafficLoad }(M)
$$

\section{b. To calculate the Node Traffic}

In EL-RPL, node traffic calculates from children count of the respective parent node

$$
\text { Node_TrafficLoad }(M)=\sum_{i=1}^{n} \text { children_count }
$$

\subsubsection{Battery Depletion Index (BDI)}

Battery depletion Index (BDI) indicates that how much percentage of energy depleted from battery present in the node. The residual energy calculates from initial energy and remaining energy of the node [4]. The residual energy calculates from equation (5).

$$
R E R(M i)=\frac{\text { Eremaining }}{\text { Einitial }}
$$

The residual energy is a remaining energy in the node $\mathrm{Mi}$ and it is represented in terms of 0 to 1 . The BDI calculation is calculated from Equation (6).

$$
B D I(M i)=(1-R E R(M i))
$$

The BDI follows the puductive rule and BDI of Path Px calculates from Equation (7).

$$
B D I(P x)=\prod_{i=1}^{n} B D I(M i)
$$

\subsection{Objective Function (OFEL)}

In DODAG, the parent selection is based on DODAG rank. The DODAG rank calculates from Min hop rank increase and objective function. The objective function takes into account the following issues such as loop freeness, data load in upward and downward routing and bottle neck near sink node. The proposed EL-RPL is evaluated the performance and fine tuned the weight values. Moreover, it provides the better efficiency, where the weight values are $\mathrm{w} 1, \mathrm{w} 2$ and $\mathrm{w} 3=1 / 3$.

$$
\min \text { OF }(\mathrm{LB}, \mathrm{BDI}, \mathrm{ETX})=\mathrm{w} 1 \times \operatorname{Load}(\mathrm{Pi})+\mathrm{w} 2 \times \mathrm{BDI}(\mathrm{Pi})+\mathrm{w} 3 \times \mathrm{ETX}(\mathrm{Pi})
$$

\subsection{Rank Calculation}

In EL-RPL, DODAG rank calculates from parent rank and rank increase value. The rank increase calculates from step value and MinHopRankIncrease. The MinHopRankIncrease default value is 256 [7]. The step value calculates from objective function and it is denoted in Equation (8). The rank calculates from Equation (9-12).

$$
\text { Step }=\mathrm{w} 1 \times \operatorname{Load}(\mathrm{Pi})+\mathrm{w} 2 \times \mathrm{BDI}(\mathrm{Pi})+\mathrm{w} 3 \times \operatorname{ETX}(\mathrm{Pi})
$$


Fixed_Point or Rank_increase $=$ Step + MinHopRankIncrease

Rank $(\mathrm{N})=$ Rank (Parent_Node) + Rank_increase

Finally, the Rank calculation based on the below equation (12)

$$
\operatorname{Rank}(\mathrm{N})=\text { floor }(\operatorname{Rank}(\mathrm{N}) / \operatorname{MinHopRankIncrease})
$$

For Instance, we have considered 6 nodes in the DODAG and each node contains Load, BDI and ETX of value. We considered that root node as "A" and its rank as 1 . Followed by, calculate the rank of Node "B and C" based on the fixed point value and Step value. Node "B and C" are bottle neck node, because it is near to sink node. EL-RPL balances the load and energy in the networks. So it avoids the early battery depletion than other similar RPL protocol. The node "D and E" calculates the rank. The participant node "F" wants to join in the existing DODAG and it has two possibilities either E or C. According to ELRPL, the DODAG computes the rank and it is decided to select node " $C$ " as parent node.

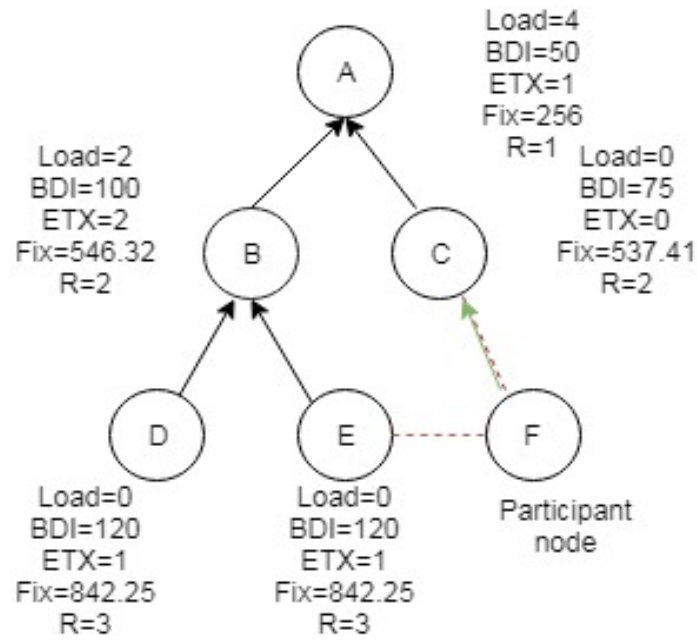

Figure 2. Rank caculation represention

The proposed EL-RPL rank calculation process is discussed in below the Table 1.

Table 1. EL=RPL Rank Calculation Process

\begin{tabular}{ccccc}
\hline Node Id & Fixed Point Value & Step Value & Rank increase & Rank \\
\hline A & 256 & 68.64 & 324 & $\mathrm{R}=$ floor(324/256) $=1$ \\
B & 546.32 & 34.32 & 290.32 & $\mathrm{R}=$ floor(546.32/256)=2 \\
C & 537.41 & 25.41 & 281.41 & $\mathrm{R}=$ floor(537.41/256)=2 \\
D & 842.25 & 39.93 & 295.93 & $\mathrm{R}=$ floor(842.25/256)=3 \\
E & 842.25 & 39.93 & 295.93 & $\mathrm{R}=$ floor(842.25/256)=3 \\
\hline
\end{tabular}

\subsection{Parent Selection Process}

In DODAG, request process is carried out in two ways. i). the participant node sends the DODAG Information Solicilisation (DIS) message to DODAG. ii). DODAG sends the DODAG Information Object (DIO) message to all participant node in periodic manner. If the participant node accepts DIO request and it wants to join in the existing DODAG, it sends the DAO control message to the parent node in DODAG. Finally, the DODAG sends the DODAG Advertisement Object-Acknowledgement (DAO-ACK) to the respective participant node. Then, the request and response based on the trickle timer interval. If the trickle time expires, the DODAG resends the DIO control messages to all participant nodes and the process is carried out the above in the same. The parent selection algorithm is given below. 


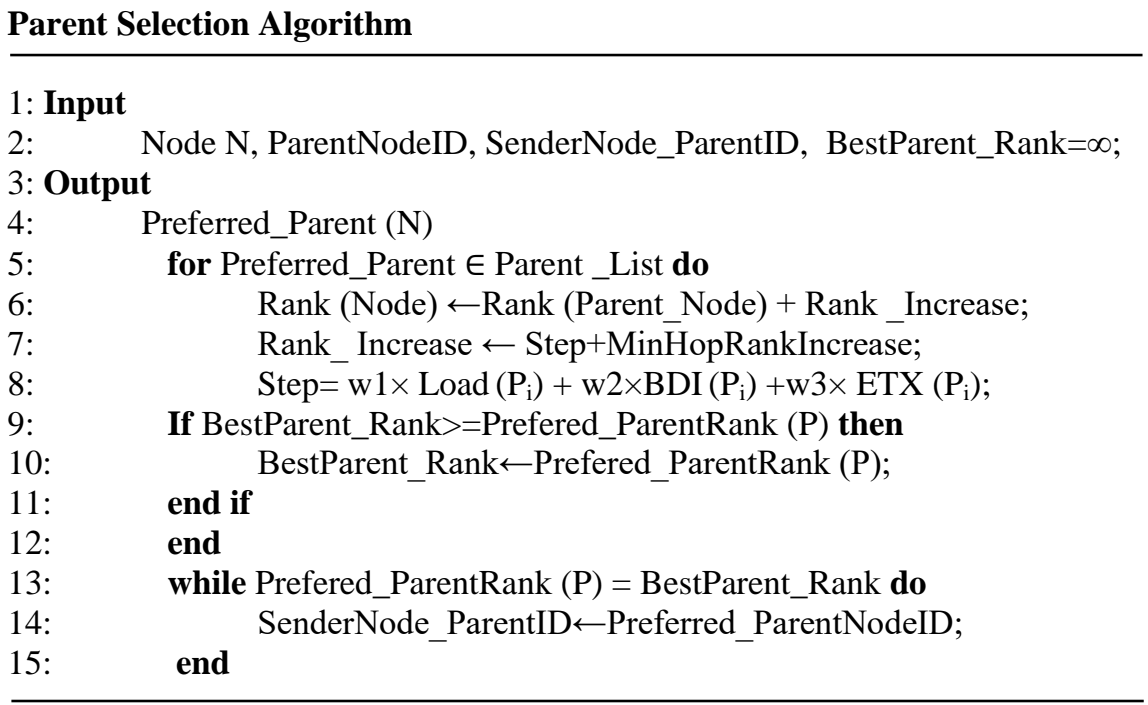

\section{PERFORMANCE EVALUATION}

We conducted the simulation of EL-RPL and it is an advancement of the standard version of RPL protocol. EL-RPL follows the additive property and it is evaluated the performance in terms of network lifetime, number of parent changes, remaining battery power, packet delivery ratio, number of parent changes and end-to-end delay. The simulation is conducted using COOJA network simulator. It is an open source operating system and it specifically designed for IoTdevices [19]. The simulation parameters represented in table-2. In MAC layer, RPL uses the default radio duty cycling mechanism and EL-RPL avoids the multi point -to-point data traffic in upward routing [20].

Most of the cases, the composite metric provides the better performance against single metric in RPL. Hence, the performance of EL-RPL compared with RER(BDI) RPL, OF-FL RPL. We conducted the simulation and it fine-tunes the weight values of the EL-RPL protocol.

Table 2. Simulation Configuration for Experiments

\begin{tabular}{ll}
\hline \multicolumn{1}{c}{ Node Id } & \multicolumn{1}{c}{ Fixed Point Value } \\
\hline Operating System & Contiki 2.7 \\
Node Type & Tmote sky \\
Minimum DIO interval & 12 \\
DIO interval doubling & 10 \\
Routing Protocol & RPL \\
MAC/Adaptation Layer & ContikiMAC/6LowPAN \\
Radio Environment & Unit Disk Graph Medium (UDGM) \\
Number of Nodes & 30 \\
Simulation Duration & $120 \mathrm{Hrs}$ \\
Full Battery & $3000 \mathrm{~mJ}$ \\
Transmission Range & $400 * 400 \mathrm{~m} 2$ \\
Data Packet Timer & $60 \mathrm{sec}$ \\
RPL Parameter & MinHopRankIncrease=256 \\
\hline
\end{tabular}

\subsection{Performance Evaluation Metrics}

EL-RPL Performance is analyzed and evaluated the following metrics.

a. Number of parent changes: It indicates parent changes in a DODAG at given amount of time.

b. Network lifetime: Network lifetime as the duration of time until the first node fails in a network due to the battery exhaustion.

c. Packet delivery ratio: Packet delivery ratio denotes that number of packets received successfully at receiver and number of packets send by sender.

d. Remaining energy: It indicates the average remaining energy present in all nodes of network.

e. End-to-end delay: It indicates the duration between start to transmit the data packet and it received by the DODAG root. 
f. Number of hopcount: It represents that the number of jump or hopcount from parent node to DODAG root.

\subsection{Evaluation Results}

We conducted the simulation and evaluate the performance of EL-RPL with RER(BDI) and composition routing metric using lexicographic and additive property.

\subsubsection{Average Number Of Parent Changes}

We measured an average number of parent changes in EL-RPL and it is compared with other similar RPL protocol such as RER(BDI) RPL and OF-FL RPL. Fig.3 shows that the number of parent changes with different composite metric based RPL protocol. X-axis indicates the different composite metric based RPL protocol and $\mathrm{Y}$-axis indicates the average number of parent changes. The average number of parent changes in RER (BDI) RPL, OF-FL RPL and EL-RPL are 0.29, 0.27 and 0.25 respectively. The number of parent changes value indicates the network stability and it is considered the lower value for stability network. However, EL-RPL provides the better network stability than RER (BDI) RPL and OF-FL RPL.

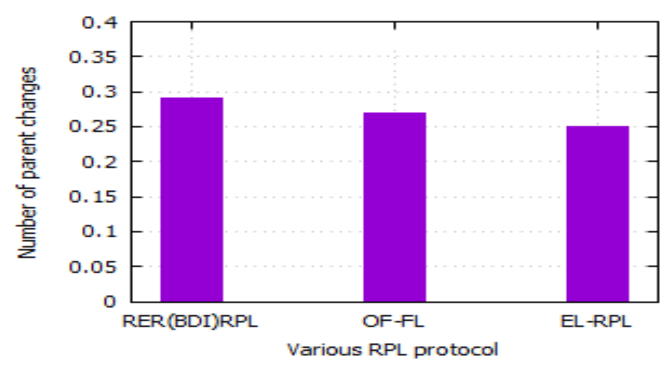

Figure 3. Various RPL protocol vs. Average number of parent changes

\subsubsection{Network Life Time}

In this scenario, we considered a DODAG consists of 1 DODAG root and 30 RPL router or host node. Through our simulation, we observed the node remaining energy of the entire node in a network. The EL-RPL network lifetime is compared with RER(BDI) RPL and OF-FL RPL. The entire simulation is conducted up to 120 hours. Fig. 4 shows that the average remaining energy of the network nodes. In this simulation, we have set the throughput as 1 packet per minute. After the simulation, the total number of alive node count as RER(BDI) RPL is 24, OF-FL RPL is 26 and EL-RPL is 27.In EL-RPL, the remaining energy distribution ratiocontains1 node have a remaining energy between 10-15\%, 7 nodes have remaining or current energy between 15-20\%, 8 nodes have remaining or current energy between 20-25\%, 7 nodes have remaining or current energy between $25-30 \%$ and 4 nodes have remaining or current energy between 30 $35 \%$. In EL-RPL, Load is one of the routing metric and it avoids the data traffic over the network. So ELRPL improves the network lifetime against RER(BDI) RPL and OF-FL RPL.

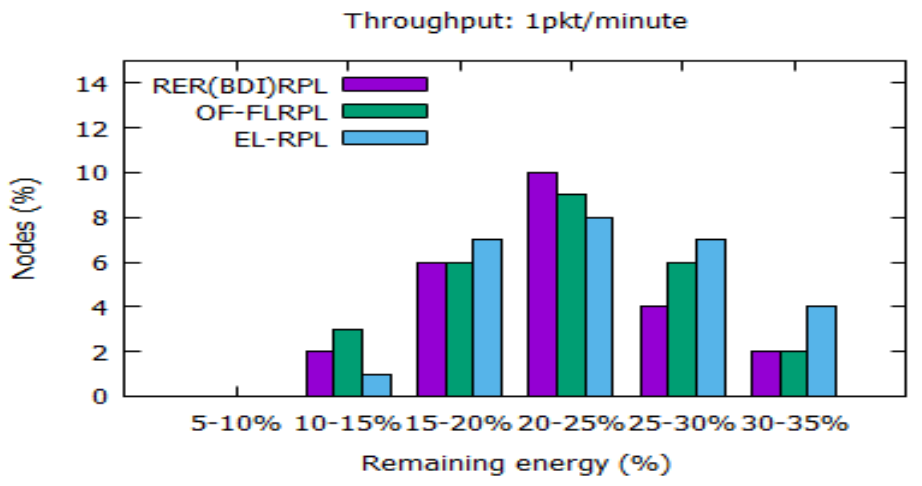

Figure 4. Comparison of remaining energy vs Number of node 


\subsubsection{Average Packet Delivery Ratio}

Packet delivery ratio indicates the relaiblity of the node present in the network. X-Axis indicates the $\mathrm{RX}$ ratio and $\mathrm{Y}$-Axis indicates the packet delivery ratio (PDR).In this simulation, we take into account the Transmit ratio (TX) value is 100 and Receive ratio (RX) is dynamically changed. Fig. 5 shows that packet delivery ratio with respect to different RX value. The proposed EL-RPL protocol PDR value compared between RER(BDI) RPLand OF-FL RPL. RER(BDI) indicates that packet delivery ratio values are 60, 63, 72, 83, 87,89 and 91 for the RX values 40, 50,60,70,80,90 and 100 respectively. OF-FL RPL indicates that packet delivery ratio values are 64,65,74,83, 89,90 and 92 for the RX values 40, 50,60,70,80,90 and 100 respectively. Likewise, EL-RPL indicates that packet delivery ratio values are 68,68,80,84, 91,93 and 95 for the RX values 40, 50,60,70,80,90 and 100 respectively. Finally, EL-RPL provides the better performance nearly 2-5\% against RER(BDR) RPL and OF-FL RPL.

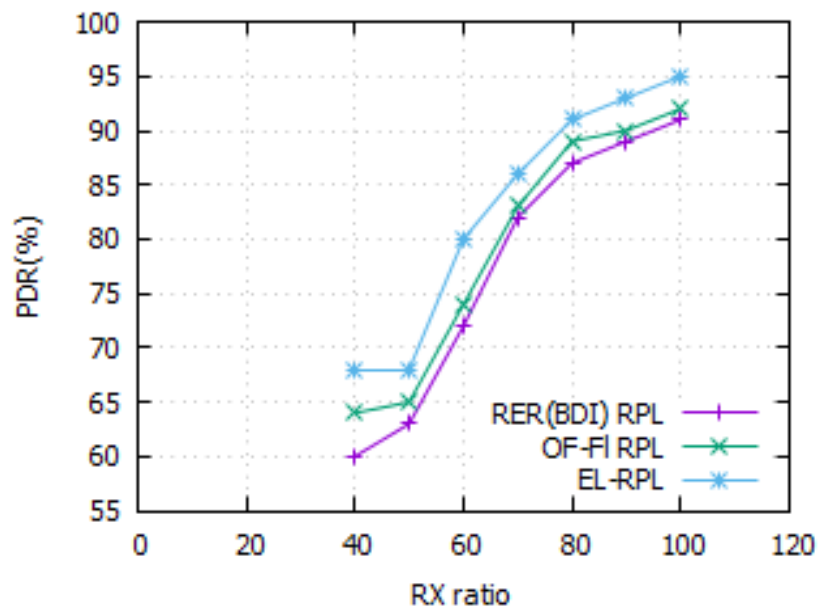

Figure 5 Packet delivery ratio vs. RX ratio

\subsubsection{Average End-to-End Delay}

The end-to-end delay is calculated from number of hop count from parent node to the DODAG root node. The delay is measured the time between sender sends the data and receiving the acknowledgement from receiver.

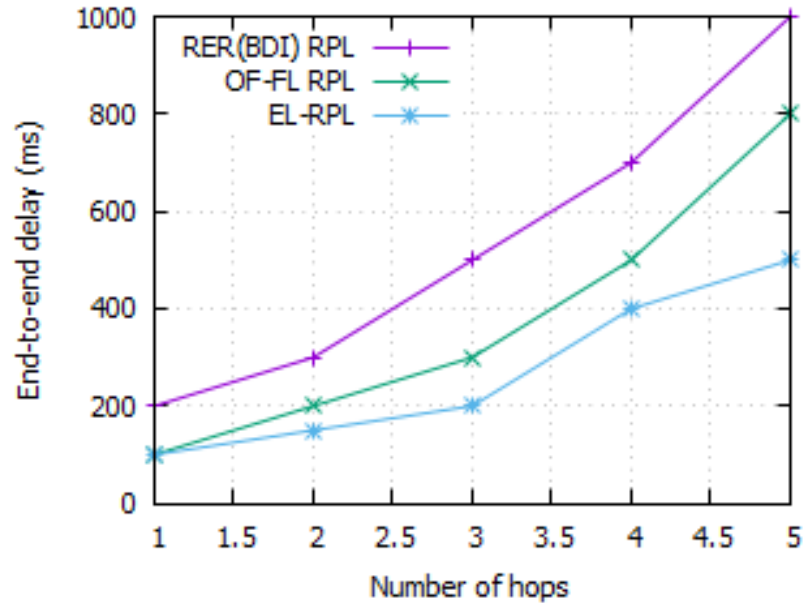

Figure 6 Number of hops vs. Average end-to-end delay (ms) 
Figure 6 shows that the average end-to-end delay of RER(BDI) RPL, OF-FL RPL and EL-RPL. This work is considered the average end-to-end delays undertake $1 \mathrm{sec}$. Neverthless, load is a prominent metric in EL-RPL and it reduces the average end-to-end delay over the network. Moreover, the figure shows that EL-RPL has less latency and it compared with RER(BDI) RPL and OF-FL RPL. EL-RPL keeps the maximum delay undergo a data packet is $0.5 \mathrm{sec}$ vs. 1sec for RER(BDI) RPL .

\subsubsection{Average End-to-End Delay}

Figure 7 shows that the comparison of average hop count in RER(BDI) RPL, OF-FL RPL and ELRPL. X-axis indicates network size and Y-axis indicates average number hop count. In EL-RPL, we used load is one of the metric and it is calculated from cumulative children count from parent node to DODAG root. However, EL-RPL allows lower hop count than RER(BDI) RPL and OF-FL RPL. In our simulation, the maximum hop count is 5 among the 30 nodes present in the network. In EL-RPL, the network size is 30 and number of hop count is 3 vs. 5 hop count for RER(BDI) RPL. Finally, EL-RPL reduces the number of hop count within the DODAG

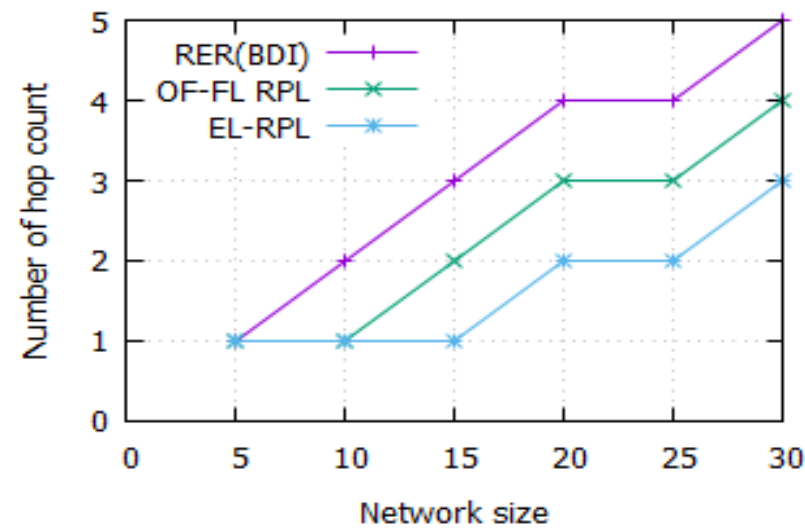

Figure 7 Network size vs. Average number of hop count

\section{CONCLUSION}

In this paper, we proposed a new energy and load aware routing protocol (EL-RPL). It deploys an objective function, which considers the metrics load, BDI and ETX, to calculate the DODAG rank, where other existing work does not consider the load metric in combination with ETX and BDI.The route with minimum value for the objective function will be the shorter route with less traffic, which is selected to send the data to DODAG root.Using the COOJA simulator, we compared the performance of EL-RPL with RERBDI RPL and OF-FL RPL. The simulation result shows that EL-RPL provides the better performance in terms of network lifetime, packet delivery ratio and end-to-end delay compared to RERBDI RPL and OF-FL RPL.

As part of future work, it is planned to apply mobility to the nodes in Low Power and Lossy Networks (LLN) and deploy it in real time environment.

\section{REFERENCES}

[1] A. Al-Fuqaha, M. Guizani, M. Mohammadi, M. Aledhari, M. Ayyash, Internet of things: A survey on enabling technologies, protocols, and applications, IEEE Communications Surveys \& Tutorials 17(2015), 2347-76.

[2] Sankar, S., and P. Srinivasan, Internet Of Things (Iot): A Survey On Empowering Tsechnologies, Research Opportunities And Applications, International Journal of Pharmacy \& Technology 8(2016), 26117-26141.

[3] Gaddour, Olfa, Anis Koubâa, and Mohamed Abid. "Quality-of-service aware routing for static and mobile ipv6based low-power and lossy sensor networks using RPL." Ad Hoc Networks 33 (2015): 233-256.

[4] Hassan, Ali, Saleh Alshomrani, Abdulrahman Altalhi, and Syed Ahsan, Improved routing metrics for energy constrained interconnected devices in low-power and lossy networks, Journal of Communications and Networks 18 (2016), 327-332.

[5] Bhattacharjee, Sanghita, and Subhansu Bandyopadhyay. "Interference aware energy efficient multipath routing in multihop wireless networks." Journal of High Speed Networks 20.4 (2014): 263-276. 
[6] Tripathi, Joydeep, Jaudelice Cavalcante de Oliveira, and Jean-Philippe Vasseur, A performance evaluation study of rpl: Routing protocol for low power and lossy networks, Information Sciences and Systems (CISS), 44th Annual Conference on. IEEE, (2010).

[7] T. Winter, P. Thubert, A. Brandt, J. Hui, R. Kelsey, P. Levis, K. Pister, R. Struik, JP. Vasseur and R. Alexander, RPL: IPv6 Routing Protocol for Low-Power and Lossy Networks, RFC6550, 2012.

[8] Zahariadis, Theodore. "Design guidelines for routing metrics composition in LLN." (2011).

[9] Iova, Oana, Fabrice Theoleyre, and Thomas Noel. "Improving the network lifetime with energy-balancing routing: Application to RPL." Wireless and Mobile Networking Conference (WMNC), 7th IFIP. IEEE, 2014.

[10] Kamgueu, Patrick Olivier, et al. Energy-based routing metric for RPL. Diss. INRIA, 2013.P. Karkazis, T. Panagiotis, C.L. Helen, S. Lambros, P. Ioannis and Z. Theodore, Design of primary and composite routing metrics for RPL-compliant Wireless Sensor Networks, Telecommunications and Multimedia (TEMU), International Conference on. IEEE, 2012.

[11] P. Karkazis, T. Panagiotis, C.L. Helen, S. Lambros, P. Ioannis and Z. Theodore, Design of primary and composite routing metrics for RPL-compliant Wireless Sensor Networks, Telecommunications and Multimedia (TEMU), International Conference on. IEEE, 2012.

[12] Kim, Hyung-Sin, et al. "Load balancing under heavy traffic in RPL routing protocol for low power and lossy networks." IEEE Transactions on Mobile Computing 16.4 (2017): 964-979.

[13] Zhu, Yanmin, et al. "On deploying relays for connected indoor sensor networks." Journal of Communications and Networks 16.3 (2014): 335-343.

[14] D.S. De Couto , D. Aguayo , J. Bicket, R. Morris, A high-throughput path metric for multi-hop wireless routing, Wireless Networks 11 (2005), 419-434.

[15] M. Qasem, A.Al-Dubai, I.Romdhani, B.Ghaleb and W.Gharibi., A new efficient objective function for routing in Internet of Things paradigm, Standards for Communications and Networking (CSCN), IEEE Conference on. IEEE, 2016.

[16] Ali, Hazrat., A performance evaluation of rpl in Contiki, (2012).

[17] F. Osterlind, A. Dunkels, J. Eriksson, N. Finne and T. Voigt, Cross-level sensor network simulation with cooja, Local computer networks, proceedings 31st IEEE conference on. IEEE, (2006). 